\title{
Refining BERT Embeddings for Document Hashing via Mutual Information Maximization
}

\author{
Zijing Ou${ }^{1}$, Qinliang Su${ }^{1 *}$ Jianxing $\mathbf{Y u}^{2}$, Ruihui $\mathbf{Z h a o}^{3}$, \\ Yefeng Zheng ${ }^{3}$, and Bang Liu ${ }^{4}$ \\ ${ }^{1}$ School of Computer Science and Engineering, Sun Yat-sen University, Guangzhou, China, \\ ${ }^{2}$ School of Artificial Intelligence, Sun Yat-sen University, Guangdong, China, \\ ${ }^{3}$ Tencent Jarvis Lab, ${ }^{4}$ RALI \& Mila, Université de Montréal, \\ ouzj@mail2.sysu.edu.cn, \{ suqliang, yujx26\}@mail.sysu.edu.cn, \\ \{zacharyzhao, yefengzheng\}@ tencent.com, bang.liu@umontreal.ca
}

\begin{abstract}
Existing unsupervised document hashing methods are mostly established on generative models. Due to the difficulties of capturing long dependency structures, these methods rarely model the raw documents directly, but instead to model the features extracted from them (e.g. bag-of-words (BOW), TFIDF). In this paper, we propose to learn hash codes from BERT embeddings after observing their tremendous successes on downstream tasks. As a first try, we modify existing generative hashing models to accommodate the BERT embeddings. However, little improvement is observed over the codes learned from the old BOW or TFIDF features. We attribute this to the reconstruction requirement in the generative hashing, which will enforce irrelevant information that is abundant in the BERT embeddings also compressed into the codes. To remedy this issue, a new unsupervised hashing paradigm is further proposed based on the mutual information (MI) maximization principle. Specifically, the method first constructs appropriate global and local codes from the documents and then seeks to maximize their mutual information. Experimental results on three benchmark datasets demonstrate that the proposed method is able to generate hash codes that outperform existing ones learned from BOW features by a substantial margin. ${ }^{1}$
\end{abstract}

\section{Introduction}

With the explosion of information, similarity search (Jing and Baluja, 2008) plays a increasingly important role in modern information retrieval systems. Traditional search engines conduct query by evaluating the distances of items in the continuous

\footnotetext{
${ }^{*}$ Corresponding author. Qinliang $\mathrm{Su}$ is also affiliated with (i) Guangdong Key Lab. of Big Data Analysis and Processing, Guangzhou, China, and (ii) Key Lab. of Machine Intelligence and Advanced Computing, Ministry of Education, China.

${ }^{1}$ Our code is available at https://github.com/J-zin/DHIM.
}

Euclidean space, making it suffer from high computational complexity and footprint. To address this issue, considerable efforts have been devoted to semantic hashing (Salakhutdinov and Hinton, 2009), which aims to represent each document by a compact binary code. Such representations are able to reduce the memory footprint and increase the retrieval efficiency significantly by enrolling in binary Hamming space.

A pivotal challenge in learning high-quality hash codes is how to retain the semantic similarities among documents. Although using supervised information is an efficient way to achieve this goal, due to the high cost of labeling, unsupervised hashing is more favourable in practice. Currently, most of unsupervised document hashing methods are established upon the perspective of deep generative models (Kingma and Welling, 2013; Rezende et al., 2014). Essentially, all these methods seek to model the documents with a deep generative model and then employ the latent representations of documents to construct hash codes (Chaidaroon and Fang, 2017; Shen et al., 2018; Dong et al., 2019; Ye et al., 2020; Zheng et al., 2020; Ou et al., 2021). Although great successes have been observed in these methods, due to the difficulties in capturing the long dependency structures of words (especially for long documents), all of these methods are established on modeling the BOW or TFIDF features of documents.

Although the BOW or TFIDF features are informative and are prevalent in many areas, their limitations are also obvious for not considering the word order and dependency structure. Recently, large-scale pre-trained language models like BERT (Devlin et al., 2018) have demonstrated their superior capabilities on various natural language understanding tasks. Embeddings extracted from them have also been shown to contain much more abundant information. Thus, in this paper, we argue that capitalizing on BERT embeddings to produce hash 
codes is better than on the out-of-date BOW features. As a first try, we modify existing generative hashing methods to accommodate the BERT embeddings and then use the trained model to generate hash codes. However, experimental results show no improvement on the quality of obtained hash codes. Even worse, the codes sometimes perform even inferior to those learned from BOW features. We conjecture that this is because the reconstruction requirement in generative hashing enforces most of the information in BERT embeddings to be transferred into the hash codes. However, as the information contained in BERT embeddings is very abundant, with only a small proportion relevant to hashing, it is not surprising to see that the codes are not aligned well with the semantic similarities of documents.

To generate high-quality hash codes from BERT embeddings, it becomes necessary to refine the embeddings to highlight the information relevant to hashing tasks (i.e., semantic information), while attenuating the irrelevant. Recent progresses on image representation learning have shown that it is possible to learn discriminative semantic representations using the mutual information (MI) maximization principle. Inspired by this, rather than utilizing the reconstruction structure, an alternative paradigm is proposed for unsupervised document hashing based on the MI maximization principle, named Deep Hash InfoMax (DHIM). The essential idea behind our approach is to construct appropriate global and local codes and then seek to maximize their mutual information, with the global and local codes accounting for the entire document and text fragments, respectively. As explained in image representation learning, doing so implicitly encourages the global codes to retain high-level semantic information shared across different local fragments, while ignoring the local irrelevant details. Extensive experiments are conducted on three benchmark datasets. The results demonstrate that by effectively refining the BERT embeddings via MI maximization principle, the proposed method is able to generate hash codes that outperform existing ones learned from BOW features by a substantial margin.

\section{Preliminaries on Generative Hashing for Documents}

Document hashing aims to learn close binary codes for semantically similar documents. An intuitive idea towards this goal is to encourage hash codes preserving as much information of documents as possible so that close codes are easier to be obtained for similar documents. Based on this idea, many methods have be proposed to employ generative models like VAEs to model the documents and then leverage the documents' latent representations to produce binary hash codes. However, due to the difficulties in capturing the long dependency structures of words (especially for long documents), existing generative hashing methods rarely seek to model the documents directly, but instead to first extract representative features from documents (e.g., BOW or TFIDF) and then perform modeling on the extracted features. Specifically, by representing a document $x$ as a sequence of words $x=\left\{w_{1}, w_{2}, \ldots, w_{|x|}\right\}$, existing generative hashing methods (Chaidaroon and Fang, 2017) are mostly established on the following document model

$$
p(x, z)=\prod_{w_{i} \in x} p_{\theta}\left(w_{i} \mid z\right) p(z),
$$

where

$$
p_{\theta}\left(w_{i} \mid z\right) \triangleq \frac{\exp \left(z^{T} E w_{i}+b_{i}\right)}{\sum_{j=1}^{|V|} \exp \left(z^{T} E w_{j}+b_{j}\right)} .
$$

Here $z$ is the latent variable; $w_{j}$ is a $|V|$ dimensional one-hot vector corresponding to the $j$-th word; $E \in \mathbb{R}^{m \times|V|}$ represents the learnable embedding matrix; $b_{i}$ is the biased term; and $|V|$ and $|x|$ represent the vocabulary size and document length, respectively. The whole model is trained by maximizing the evidence lower bound (ELBO) of log-likelihood

$$
\mathcal{L}(\theta, \phi)=\mathbb{E}_{q_{\phi}(z \mid x)}\left[\log \frac{p_{\theta}(x, z)}{q_{\phi}(z \mid x)}\right]
$$

with respect to $\theta$ and $\phi$, where $q_{\phi}(z \mid x)$ denotes the approximate posterior distribution parameterized by $\phi$. After training, representation of the document $x$ can be extracted from the approximate posterior $q_{\phi}(z \mid x)$, e.g., using its output mean. Note that a simple decoder of (2) is adopted purposely for better transferring similarity information of documents $x$ into the latent representations $z$.

In the early generative hashing work VDSH (Chaidaroon and Fang, 2017), Gaussian distributions are employed for both the prior $p(z)$ and approximate posterior $q_{\phi}(z \mid x)$ directly. But due to 
the continuous characteristics of Gaussian random variables, a separate binarization step is required to transform the continuous latent representations into binary codes. To overcome the separate training issue, Bernoulli prior and posterior are then proposed in NASH (Shen et al., 2018). With the recent advances on gradient estimators for discrete random variables, the model successfully circumvents gradient backpropagation issue for discrete variables, and can be trained efficiently in an end-to-end manner. Inspired by NASH, many variant methods are then proposed by using more sophisticated prior or posterior distributions, with the objective to model the documents more accurately, such as Bernoulli mixture prior in BMSH (Dong et al., 2019) and Boltzmann machine posterior in CorrSH (Zheng et al., 2020) etc.

Despite of the observed remarkable performance, all of the methods mentioned above rely on the document model (1), which, however, is essentially established on the BOW features of documents, without considering any word order and dependency information. Although BOW features are informative, their limitations are also obvious due to the neglect of word order and dependency structure. With the development of large-scale pre-trained models like BERT, it becomes easy to obtain semanticsrich features that contain long dependencies and contextual information. Thus, we argue that it is beneficial to capitalize on the information-rich BERT embeddings over the out-of-date BOW features to learn hash codes.

\section{Hashing on BERT Embeddings via Generative Models}

Feeding a document $x=\left\{w_{1}, w_{2}, \cdots, w_{|x|}\right\}$ into a pre-trained BERT model could produce an embeding/feature for the document, denoted as $\mathcal{B}(x)$ for subsequent presentation. Inspired by the success of generative hashing methods, we modify them to accommodate the BERT embeddings. Due to the difference between BERT embeddings and BOW features, the decoder in (2) is replaced by a conditional Gaussain distribution

$$
p_{\theta}(x \mid z)=\frac{1}{\left(2 \pi \sigma^{2}\right)^{d / 2}} e^{-\frac{\|\mathcal{B}(x)-W z\|^{2}}{2 \sigma^{2}}},
$$

where $W$ is the learnable model parameter and the bias term is omitted for brevity; and $d$ denotes the dimension of BERT embeddings. Similar to the generative hashing models introduced above, here a simple decoder is employed purposely to facilitate the transferring of similarity information of BERT embeddings into the latent codes $z$. To achieve end-to-end training and directly output binary codes, Bernoulli prior $p(z)$ and approximate posterior $q_{\phi}(z \mid x)$ can be used, as done in NASH, BMSH etc. After training, the binary hash code of document $x$ can be obtained from the latent codes $z \sim q_{\phi}(z \mid x)$.

Unexpectedly, as observed in experiments (see Table 2), the codes generated from BERT embeddings in this manner perform even worse than that from TFIDF features. At first glance, this is unreasonable, since information in BERT embeddings is much abundant. However, we ought to emphasize that more information does not represent better performance. Although the BERT embedding has been successfully applied to various downstream tasks, it is also reported that directly using BERT embeddings can not yield satisfactory gains to information retrieval (Reimers and Gurevych, 2019). Li et al. (2020) attributed this issue to that the embedding contains many types of information, and the semantic information is not appropriately preserved. In this regard, the worse performance of naively exploiting BERT embeddings is traceable. In the generative hashing approach, what the model does basically is to compress the embedding $\mathcal{B}(x)$ into a latent code $z$ and then use the code to reconstruct the original embedding $\mathcal{B}(x)$. Due to the requirement of reconstruction, latent codes $z$ are enforced to preserve as much information of original inputs $\mathcal{B}(x)$ as possible. However, as discussed above, BERT embeddings contain various kinds of information, and the categorical information is just the one relevant to the hashing performance while the others are redundant. Thus, when the generative approach is applied to BERT embeddings, it is not surprising to see that the codes are not aligned well with the semantic similarities of documents.

\section{Refining BERT Embeddings via MI Maximization}

According to discussions in Section 3, to produce high-quality hash codes, it is necessary to refine BERT embeddings to highlight the categoryrelevant information, while attenuating the other types of information. Recent progresses on image representation learning (Hjelm et al., 2018) have demonstrated that it is possible to learn categorydiscriminative representations from images unsu- 
pervisedly with the MI maximization principle. Inspired by this, a brand new hashing framework based on MI maximization principle is proposed, which learns binary hash cods from BERT embeddings without using the reconstruction requirement, thereby overcoming the issues associated with the generative hashing approaches.

\subsection{Deep InfoMax Review}

Deep InfoMax (Hjelm et al., 2018) learns categorydiscriminative representations for images by maximizing the mutual information between global and local representations. It first constructs a global representation for an image and lots of local representations, both extracted from the image's CNN feature maps. Then, it estimates the MI between the global and local representations and maximizes it. As explained in (Hjelm et al., 2018), since there are many local representations and each of them accounts for a local region of an image, maximizing the global-local MI implicitly encourages the global representation to retain global semantic information that is shared across all local regions, while ignoring specific details exclusive to different local regions.

\subsection{Construction of Global/Local Document Features}

To refine BERT embeddings with the deep InfoMax, we first need to construct appropriate global and local document features. To this end, we rerepresent a document as $X=\left\{e_{1}, \ldots, e_{T}\right\}$, where $e_{i} \in \mathbb{R}^{d}$ is the BERT embedding of the $i$-th word in the document, and $T$ denotes the document length. Then, we pass the document $X$ through a textual CNN (Kim, 2014), in which filters $W \in \mathbb{R}^{K \times n \times d}$ are convolved with the words sequentially, with $n$ and $K$ denoting the filter size and number, respectively. Obviously, such operation could generate local features for every piece of $n$-gram fragments. Specifically, the local feature for the $i$-th fragment is computed as

$$
h_{i}^{(n)}=\operatorname{Re} L U\left(W * e_{i: i+n-1}\right),
$$

where $*$ denotes the convolution operator, and the bias term is omitted for brevity; and $\operatorname{Re} L U(\cdot)$ represents the rectified linear unit (ReLU) function. By applying this filter to all text fragments, we obtain the local feature maps at all locations

$$
H^{(n)}=\left\{h_{1}^{(n)}, h_{2}^{(n)}, \ldots, h_{T}^{(n)}\right\} .
$$



Figure 1: Architecture of the DHIM, in which W.S.n denotes convolution operation with window size $n$.

By passing $H^{(n)}$ to READOUT function, which can be a simple mean-over-time pooling operation (Collobert et al., 2011) or more sophisticated selfattention mechanism (Vaswani et al., 2017), we obtain the document's global feature.

To further highlight the semantic information in global features, we propose to compute multigranularity local and global features using different window sizes of convolution operation (set as $\{1,3,5\}$ in our experiments). That is, the final local and global features are computed as

$$
\begin{aligned}
h_{i} & =\operatorname{MLP}\left(\operatorname{CONCAT}\left(\left\{h_{i}^{(n)}\right\}_{n \in \mathcal{N}}\right)\right), \\
H & =\operatorname{READOUT}\left(\left\{h_{i}\right\}_{i=1}^{T}\right),
\end{aligned}
$$

where $\mathcal{N}$ denotes the set of different window sizes and MLP is the multilayer perception layer used to project the feature maps on desirable dimension. By maximizing the MI between global and local document features, the global feature $H$ is encouraged to keep high-level semantic information that are shared across all local fragments, while ignoring the irrelevant local details.

\subsection{End-to-End Hashing by Maximizing the Global-Local MI}

Maximizing the global-local MI is able to yield semantic-rich global features $H$, which, however, are in the real-valued space. To obtain the binary hash codes, a feasible way is to binarize the global feature $H, e . g$., by setting a threshold value. Obviously, the separate binarization strategy is not optimal in producing high-quality of codes. To obtain hashing models that admit end-to-end training, inspired by end-to-end generative hashing schemes, we propose to generate binary global and local representations by adding a probabilistic Bernoulli layer, that is,

$$
\begin{aligned}
b_{i} & \sim \operatorname{Bernoulli}\left(\sigma\left(h_{i}\right)\right), \\
B & \sim \operatorname{Bernoulli}(\sigma(H)),
\end{aligned}
$$

where $b_{i}$ and $B$ denote local and global binary representations, respectively; and $\sigma(\cdot)$ denotes the 


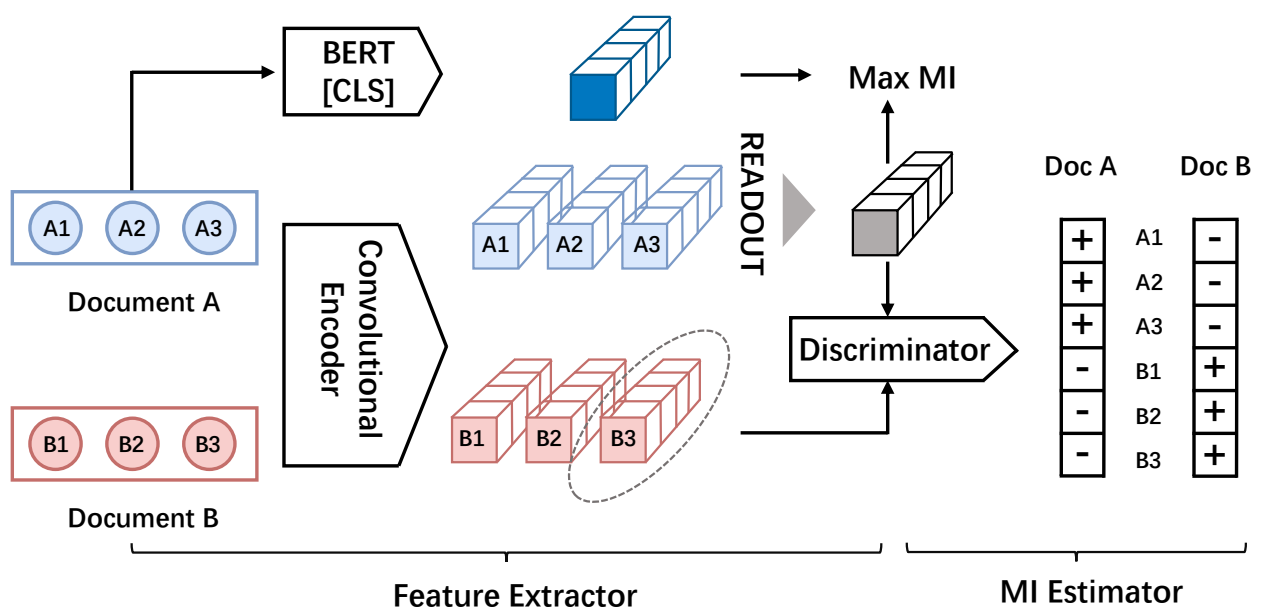

Figure 2: Intuitive illustration of DHIM. The local/global features are captured by textual convolution neural network and then fed into discriminator to identify whether they are from the same document. For example, consider a batch input with 2 documents with 3 words for each. For the global representation (gray cuboid) of document A, there will be 6 input pairs (local/global features) to the discriminator and same for document B. Additionally, we further encourage the mutual information between the learned representations and BERT CLS embedding to be high such that refining more semantic information into binary codes.

sigmoid function that transforms the features into probability. The probabilistic binarization layer allows the gradient to be estimated efficiently by backpropagation-like algorithms like ST (Bengio et al., 2013), Gumbel-softmax (Jang et al., 2016) etc., which are also widely used in the end-toend generative hashing models. The overall architecture of generating binary representations is depicted in Figure 1.

Since our goal is to learn binary hash codes, instead of maximizing the MI between $H$ and $h_{i}$, we propose to maximize the MI between the global and local binary features $B$ and $b_{i}$ directly

$$
\hat{\theta}=\underset{\theta}{\arg \max } \frac{1}{T} \sum_{i=1}^{T} I\left(b_{i} ; B\right),
$$

where $\theta$ is the model parameters involved in the construction of global and local binary representations. Note that $b_{i}$ and $B$ are not specific for one document, but for all documents in the training set. Mutual information is notoriously hard for evaluation. Recently, many sophisticated methods have been proposed to estimate it, such as MINE (Belghazi et al., 2018), infoNCE (Oord et al., 2018) and Jensen-Shannon divergence estimator (JSDE) (Nowozin et al., 2016). Among them, JSDE is known to be less sensitive to the number of negative samples, thus we apply it to estimate the MI and then optimize it w.r.t. the model paramters. Specifically, the MI can be estimated by minimiz- ing the following function w.r.t. $\phi$

$$
\begin{aligned}
\tilde{I}_{\phi}\left(b_{i} ; B\right)= & -\operatorname{softplus}\left(-D_{\phi}\left(b_{i}, B\right)\right) \\
& -\mathbb{E}_{\tilde{\mathbb{P}}}\left[\operatorname{softplus}\left(D_{\phi}\left(\tilde{b}_{i}, B\right)\right)\right],
\end{aligned}
$$

where $\tilde{b}_{i}$ is the $i$-th local representation of negative samples generated from empirical distribution $\tilde{\mathbb{P}}=$ $\mathbb{P}$; softplus function is defined as $\operatorname{softplus}(x) \triangleq$ $\log \left(1+e^{x}\right)$; and $D_{\phi}(\cdot, \cdot)$ is a discriminator realized by a neural network with parameter $\phi$. In practice, negative sample $\tilde{b}_{i}$ is chosen from local representations of other documents in a minibatch.

The MI maximization scheme above relies solely on BERT embeddings of individual words, totally ignoring the embedding corresponding to the CLS token of BERT. The CLS embeddings are known to preserve global information of sentences or documents. Thus, to improve the global semantic information in the learned codes, we add a regularization term to boost the MI between the codes and CLS embedding. Therefore, the final loss takes the form

$$
\tilde{\mathcal{L}}(\phi, \theta)=-\frac{1}{T} \sum_{i=1}^{T} \tilde{I}_{\phi}\left(b_{i} ; B\right)-\beta \tilde{I}_{\phi}(E ; B),
$$

where $\beta$ is a hyper-parameter; and $E$ denotes the binarized CLS embedding, obtained in similar way to (7). Note that $\theta$ is the model parameters involved in the construction of $b_{i}$ and $B$, while $\phi$ is used in the discriminator $D_{\phi}(\cdot, \cdot)$. By resorting to the gradient estimator for discrete random variables, the loss $\tilde{\mathcal{L}}(\phi, \theta)$ can be optimized efficiently 
with stochastic gradient decent (SGD) algorithms An overall depiction of the proposed Deep Hash InfoMax (DHIM) model is illustrated in Figure 2.

\section{Related Work}

Early works in unsupervised document hashing generally built upon the generative models (Kingma and Welling, 2013; Rezende et al., 2014), in which the encoder-decoder architecture was established to encourage binary codes to retain semantic information by reconstructing original data. For examples, VDSH (Chaidaroon and Fang, 2017) first proposed to learn continuous representations under variational autoencoder (VAE) framework, and then cast it into binary codes. However, the two-stage training procedure is prone to undermine the performance. NASH (Shen et al., 2018) tackled this issue by replacing Gaussian prior with Bernoulli in VAE and adopting straight-through to enable end-to-end training. Since then, a lot of methods surged to improve the performance.

Specifically, Dong et al. (2019) proposed to employ mixture distribution as prior to enhance model's capabilities; Ye et al. (2020) introduced auxiliary topic vectors to address the problem of information loss in few-bits scenarios, and Zheng et al. (2020) employed Boltzmann posterior to introduce correlation among bits. Beyond generative models, AMMI (Stratos and Wiseman, 2020) achieved superior performance by maximizing mutual information between documents and codes. However, the adversarial training procedure used in AMMI is extremely unstable. Although these models are impressive, one common issue of them is that they simply exploited bag-of-words features as input, which is not enough to capture the rich semantic information of documents.

Recently, information theory enables a simple and insightful paradigm of unsupervised representation learning (Oord et al., 2018; Stratos and Wiseman, 2020; Qiu et al., 2021). For example, Hjelm et al. (2018) proposed an unsupervised representation learning algorithm on image data, called Deep InfoMax, which maximizes the MI between the whole image and local patches. Velickovic et al. (2019) and Sun et al. (2019) extended this idea on graph data, in which the representations can be learned by maximizing the MI between the whole and sub graphs. These methods consistently encourage the global representations to retain similar interest of local features. Following similar ideas,

\begin{tabular}{cccccc}
\hline Dataset & Train & Val & \multicolumn{2}{c}{ Test } & \multicolumn{2}{c}{ Classes } & AvgLen \\
\hline NYT & 9,221 & 1,154 & 1,152 & 26 & 648 \\
DBpedia & 50,000 & 5,000 & 5,000 & 14 & 47 \\
AGNews & 114,839 & 6,381 & 6,380 & 4 & 32 \\
\hline
\end{tabular}

Table 1: The statistic of three benchmark datasets.

we train our models that maximize MI between local n-grams features and the pooled global document representation, which can efficiently distill the semantic information of BERT embedding into hash codes.

\section{Experiments}

\subsection{Experiment Setup}

Datasets We verify the proposed model on three public benchmark datasets: i) The New York Times (NYT) (Tao et al., 2018), which contains news articles published by The New York Times; ii) DBpedia (Lehmann et al., 2015), which contains the abstract of articles extracted from Wikipedia; iii) AGNews (Zhang et al., 2015), which is a news collection gathered from academic news search engine. For all documents in a dataset, we simply apply the same string cleaning operation ${ }^{2}$ conducted in (Kim, 2014). After that, it is randomly split into training, validation and test sets, with the statistics shown in Table 1.

Baselines We compare our model with the following unsupervised deep semantic hashing methods: VDSH (Chaidaroon and Fang, 2017), NASH (Shen et al., 2018), BMSH (Dong et al., 2019), WISH (Ye et al., 2020), CorrSH (Zheng et al., 2020) and AMMI (Stratos and Wiseman, 2020). The TFIDF features and BERT embeddings are taken as input to evaluate their impact for baselines. We exploit sklearn TfidfVectorizer API to extract TFIDF features for each document with the number of dimension in $10,000,20,000$, and 20, 000 for NYT, DBpedia and AGnews, respectively. BERT embedding is the CLS embedding, whose dimension is 768 . For all baselines, we tune their parameters on the validation set and select the best one to evaluate on the test set.

Training Details We implement our model with PyTorch and HuggingFace API (Wolf et al., 2019). In our experiment, the discriminator $D_{\phi}$ is constituted by a one-layer feed-forward neural network

\footnotetext{
${ }^{2}$ https://github.com/yoonkim/CNN_sentence
} 


\begin{tabular}{|c|c|c|c|c|c|c|c|c|c|c|c|c|}
\hline \multirow{2}{*}{ Method } & \multicolumn{4}{|c|}{ NYT } & \multicolumn{4}{|c|}{ DBpedia } & \multicolumn{4}{|c|}{ AGNews } \\
\hline & 16bits & $32 \mathrm{bits}$ & bits & 8 bits & 16bits & 32bits & 64bits & 128bits & | 16bits & $32 \mathrm{bits}$ & 64bits & 128bits \\
\hline & 7 & 0 & 1 & 0.7849 & 79 & 0.7264 & 4 & 0.8491 & $\mid 0.6732$ & 0.6742 & 0.7270 & 7386 \\
\hline $\mathrm{NASH}^{*}$ & 0.7487 & 0.7552 & .7508 & 0.7301 & 0.7802 & 0.7984 & 0.7979 & 0.7676 & 0.6574 & 0.6934 & 0.7272 & 0.7433 \\
\hline WISH ${ }^{*}$ & 0.7015 & 0.7003 & 0.6448 & 0.6894 & 0.8228 & 0.8276 & 0.8210 & 0.7822 & 0.7453 & 0.7479 & 0.7505 & 0.7270 \\
\hline $\mathrm{BMSH}^{*}$ & 0.7402 & 0.7638 & 0.7688 & 0.7763 & 0.8317 & 0.8624 & 0.8705 & 0.8386 & 0.7409 & 0.7603 & 0.7609 & 0.7356 \\
\hline CorrSH $^{\mathbf{2}}$ & .7543 & 0.7761 & 0.7724 & 0.7839 & 0.8201 & 0.8178 & 0.8094 & 0.8577 & 0.7620 & 0.7645 & 0.7661 & 0.7767 \\
\hline $\mathrm{AMMI}^{*}$ & 0.7106 & 0.7648 & 0.7737 & 0.7803 & 0.8451 & 0.8953 & 0.9078 & 0.9103 & 0.7647 & 0.7661 & 0.7732 & 0.7823 \\
\hline & & 0 & & & & & & & & & & 027 \\
\hline $\mathrm{NASH}^{\star}$ & 0.5587 & 0.5825 & 0.6098 & 0.6427 & 0.6587 & 0.7454 & 0.7796 & 0.8143 & 0.6632 & 0.6844 & 0.7040 & 0.7207 \\
\hline WISH ${ }^{\infty}$ & 0.5883 & 0.6475 & 0.6547 & 0.7034 & 0.6565 & 0.7291 & 0.7666 & 0.8229 & 0.6535 & 0.6619 & 0.6939 & 0.7203 \\
\hline $\mathrm{BMSH}^{\star}$ & 0.5935 & 0.6326 & 0.6587 & 0.6971 & 0.6642 & 0.7913 & 0.8201 & 0.8 & 0.6677 & 0.6961 & 0.7199 & 0.7316 \\
\hline CorrSH $^{\star}$ & 0.6203 & 0.6548 & 0.6838 & 0.7228 & 0.6528 & 0.7463 & 0.7865 & 0.8361 & 0.6706 & 0.6851 & 0.7086 & 0.7317 \\
\hline $\mathrm{AMMI}^{\boldsymbol{\phi}}$ & 0.6047 & 0.6510 & 0.6967 & 0.7447 & 0.8025 & 0.8267 & 0.8926 & 0.8674 & 0.6550 & 0.6826 & 0.7185 & 0.7436 \\
\hline DHIM & .7969 & 0.8055 & 7977 & . & 0.9426 & 0.9480 & 0.9302 & 0.002 & $0.102 \mathrm{~J}$ & 0.7917 & 0.7888 & 0.7986 \\
\hline
\end{tabular}

Table 2: The precision on three datasets with different numbers of bits in unsupervised document hashing. \& and \$ denote that the input document features are TFIDF and BERT embeddings, respectively.

\begin{tabular}{|c|c|c|c|c|c|}
\hline \multicolumn{2}{|c|}{ Ablation Study } & 16bits & 32 bits & 64bits & 128bits \\
\hline \multirow{3}{*}{ NYT } & DHIM $_{\text {median }}$ & 0.7040 & 0.6949 & 0.6943 & 0.6999 \\
\hline & DHIM $_{\text {w/o reg }}$ & 0.7371 & 0.7639 & 0.7704 & 0.7647 \\
\hline & DHIM & 0.7969 & 0.8055 & 0.7977 & 0.7909 \\
\hline \multirow{3}{*}{ DBpedia } & DHIM $_{\text {median }}$ & 0.7955 & 0.8432 & 0.8530 & 0.8630 \\
\hline & DHIM $_{\text {w/o reg }}$ & 0.9057 & 0.9327 & 0.9206 & 0.8788 \\
\hline & DHIM & 0.9426 & 0.9480 & 0.9302 & 0.8821 \\
\hline \multirow{3}{*}{ AGnews } & DHIM $_{\text {median }}$ & 0.7431 & 0.7538 & 0.7767 & 0.7897 \\
\hline & DHIM $_{\text {w/o reg }}$ & 0.7629 & 0.7622 & 0.7821 & 0.7944 \\
\hline & DHIM & 0.7823 & 0.7917 & 0.7888 & 0.7986 \\
\hline
\end{tabular}

Table 3: The performance of variant models of DHIM.

followed with a sigmoid activation function, and the READOUT function is simply implemented as mean-pooling. We exploit the output of BERTbase module (Devlin et al., 2018) as the features of documents. During training, the parameters of pre-trained BERT network are fixed, while only training the proposed convolutional encoder. We employ Adam optimizer for optimization (Kingma and $\mathrm{Ba}, 2014)$, with the learning rate selected from $\left\{1 \times 10^{-3}, 1 \times 10^{-4}, 1 \times 10^{-5}\right\}$, and coefficient $\beta$ from $\{0.1,0.2, \ldots, 1\}$, according to the performance observed on the validation set.

Evaluation Metrics Same as the previous works (Chaidaroon and Fang, 2017), the retrieval precision is used to measure the quality of generated hash codes. For each query document, we retrieval its top-100 most similar documents based on the Hamming distance of learned codes. Then the retrieval precision is calculated as the percentage of the retrieved documents sharing with the same label as the query. Finally, The precision averaged over the whole test set is reported as the performance of the evaluated method.

\begin{tabular}{c|cccc}
\hline Features & 16bits & 32bits & 64bits & 128bits \\
\hline Random & 0.8140 & 0.8377 & 0.8666 & 0.8612 \\
GloVe & 0.8334 & 0.8507 & 0.8734 & 0.8611 \\
BERT $_{\text {base }}$ & 0.9426 & 0.9480 & $\mathbf{0 . 9 3 0 2}$ & 0.8821 \\
BERT $_{\text {large }}$ & 0.9167 & 0.9261 & 0.9013 & $\mathbf{0 . 8 9 0 2}$ \\
ROBERTA $_{\text {base }}$ & 0.9383 & 0.9437 & 0.9142 & 0.8728 \\
ROBERTA $_{\text {large }}$ & $\mathbf{0 . 9 5 2 1}$ & $\mathbf{0 . 9 5 2 7}$ & 0.9144 & 0.8706 \\
\hline
\end{tabular}

Table 4: The performance of models with variant document features on the DBpedia datasets.

\subsection{Results and Analysis}

Overall Performance The performances of our proposed model DHIM and all baselines are demonstrated in Table 2. It can be seen that our model performs favorably to the current state-of-the-art methods, yielding best performance across different datasets and settings. Compared with taking TFIDF as input, we find that the performance declines sharply if directly taking BERT embedding as input and redefining the generative model as Gaussian. This may be attributed to the fact that the reconstruction-based models may potentially tend to pay more attention on the generation of semantically-irrelevant information. However, if further refining the BERT embeddings via the proposed DHIM model, significant performance gains can be observed, which strongly corroborates the benefit of mutual information maximization framework. When examining the performance across different code lengths, our proposed method can achieve comparable performance with short codes. This is an attractive nature, since remarkable gratuity can be acquired profitably on the short codes, which is more suitable for low resource (small footprint) scenarios. 


\begin{tabular}{c|c|c}
\hline Distance & Category & Content \\
\hline query & Athlete & Ilya Aleksandrovich Borodin (born July 6 1976) is a Russian professional footballer \\
1 & Athlete & Vojislav vodka Meli (5 January 1940 - 7 April 2006) is a former Yugoslavian footballer \\
5 & Athlete & Rik Goyito Gregorio pérez (born November 19 1989) is a Mexican mixed martial artist \\
10 & Artist & Themistocles Popa (June 27 1921 - November 26 2013) was a Romanian composer musician \\
20 & Film & Allpakallpais a 1975 Peruvian drama film directed by Bernardo Arias \\
30 & Transportation & USS Alcor (ad 34) was a destroyer tender the lone ship in her class named \\
\hline
\end{tabular}

Table 5: Qualitative analysis of the learned 32-bit hash codes on the DBpedia dataset. We present the documents with Hamming distance of $1,5,10,20$ and 30 to the query.
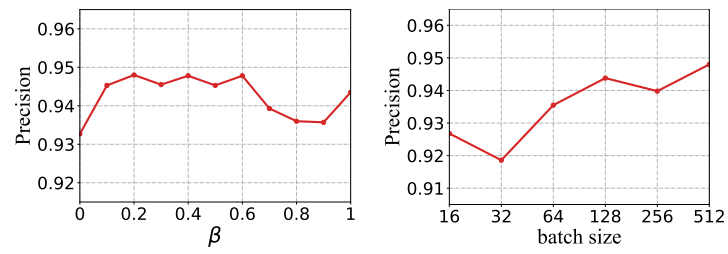

Figure 3: Parameter sensitivity analysis for the $\beta$ and batch size with 32-bit hash codes on DBpedia.

Ablation Study To understand the influence of different components of DHIM, we further experiment with two variants of our model: i)

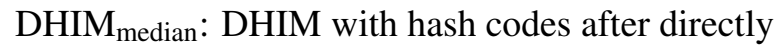
binarizing the real-value representations using the median value as the threshold; ii) DHIM $_{\mathrm{w} / \mathrm{o} \text { reg: }}$ : DHIM without semantic-preserving regularizer. As seen from Table 3, DHIM ${ }_{\mathrm{w} / \mathrm{o} \text { reg }}$ achieves better per-

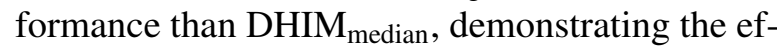
fectiveness of our proposed adaptions on the original deep InfoMax framework, i.e., introducing a probabilistic layer to enable end-to-end training. Moreover, the additional semantic-preserving regularization is benefit to integrate expressive semantic information. This can be verified by significant per-

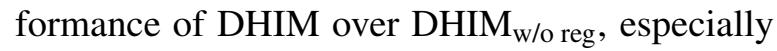
in short bits scenarios. However, the performance gap between them becomes small as code length increases. We attribute this interesting observation to the fact that the increased generalization ability of models brought by large bits is inclined to alleviate the impact of semantic regularization.

Impact of Different Features One desirable property of DHIM is that we can exploit different textual features to enhance model abilities. To understand their effects, we investigate the impact of different kinds of word features: i) Random: with randomly initialized word embeddings; ii) GloVe: with the GloVe embeddings (Pennington et al., 2014); iii) Pre-trained: with the ouputs of BERT (Devlin et al., 2018) or ROBERTA (Liu et al., 2019). As seen from Table 4, simply exploiting random embeddings, our model still achieves comparable performance, demonstrating the effectiveness

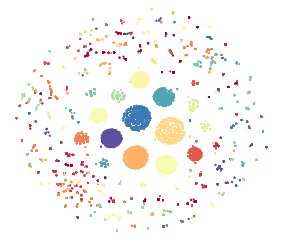

(a) DHIM

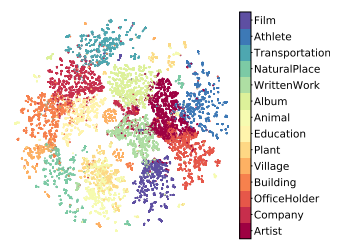

(b) AMMI
Figure 4: Visualization of the 32-bit codes learned by the proposed models for the DBpedia dataset.

of the proposed mutual information maximization based hashing framework. It is worth to note that the model trained on pre-trained features yields better performance. This proves that the expressive context information of the document is conducive to learning high-quality hash codes.

Parameter Sensitivity We also investigate the influence of hyperparameter $\beta$ and minibatch size. As shown in the left column of Figure 3, compared with the case of $\beta=0$, significant performance gains can be obtained by introducing semantic regularization. However, the appropriate value of $\beta$ should be chosen carefully, since the best performance cannot be guaranteed if $\beta$ is too small or too large. Since the number of negative samples plays important roles in MI estimation, we further investigate the impact of batch size. From the right column of Figure 3, we see that as batch size increases, the performance rises gradually and then converges to certain level.

Case Study To evaluate the quality of generated codes more intuitively, we present a retrieval case of the given query documents. As shown in Table 5, as the Hamming distance increases, the semantic of the retrieved document becomes less relevant, illustrating that the hash codes can effectively capture the semantic information.

Visualization of Hash Codes In Figure 4, we project the learned binary codes into 2-dimensional plane with t-SNE (van der Maaten and Hinton, 2008) technique. It can be seen that the codes produced by our DHIM are more distinguishable 
than those of AMMI, demonstrating the superiority of our method.

\section{Conclusion}

We have proposed an effective and efficient semantic hashing method by refining the BERT embedding. Specifically, we applied a textual convolutional neural network with probabilistic layers to capture local and global features, and refined semantic information into binary codes by maximizing their mutual information. Extensive evaluations demonstrated that our model significantly outperforms baseline methods by learning hash codes under the guidance of MMI frameworks.

\section{Acknowledgement}

This work is supported by the National Natural Science Foundation of China (No. 61806223, U1811264), Key R\&D Program of Guangdong Province (No. 2018B010107005), National Natural Science Foundation of Guangdong Province (No. 2021A1515012299), Science and Technology Program of Guangzhou (No. 202102021205). This work is also supported by Huawei MindSpore.

\section{References}

Mohamed Ishmael Belghazi, Aristide Baratin, Sai Rajeshwar, Sherjil Ozair, Yoshua Bengio, Aaron Courville, and Devon Hjelm. 2018. Mutual information neural estimation. In International Conference on Machine Learning, pages 531-540. PMLR.

Yoshua Bengio, Nicholas Léonard, and Aaron Courville. 2013. Estimating or propagating gradients through stochastic neurons for conditional computation. arXiv preprint arXiv:1308.3432.

Suthee Chaidaroon and Yi Fang. 2017. Variational deep semantic hashing for text documents. In Proceedings of the 40th International ACM SIGIR Conference on Research and Development in Information Retrieval, pages 75-84.

Ronan Collobert, Jason Weston, Léon Bottou, Michael Karlen, Koray Kavukcuoglu, and Pavel Kuksa. 2011. Natural language processing (almost) from scratch. Journal of Machine Learning Research, 12(ARTICLE):2493-2537.

Jacob Devlin, Ming-Wei Chang, Kenton Lee, and Kristina Toutanova. 2018. BERT: Pre-training of deep bidirectional transformers for language understanding. arXiv preprint arXiv:1810.04805.

Wei Dong, Qinliang Su, Dinghan Shen, and Changyou Chen. 2019. Document hashing with mixture-prior generative models. arXiv preprint arXiv:1908.11078.

R Devon Hjelm, Alex Fedorov, Samuel LavoieMarchildon, Karan Grewal, Phil Bachman, Adam Trischler, and Yoshua Bengio. 2018. Learning deep representations by mutual information estimation and maximization. arXiv preprint arXiv:1808.06670.

Eric Jang, Shixiang Gu, and Ben Poole. 2016. Categorical reparameterization with Gumbel-softmax. arXiv preprint arXiv:1611.01144.

Yushi Jing and Shumeet Baluja. 2008. VisualRank: Applying PageRank to large-scale image search. IEEE Transactions on Pattern Analysis and Machine Intelligence, 30(11):1877-1890.

Yoon Kim. 2014. Convolutional neural networks for sentence classification. In Proceedings of the 2014 Conference on Empirical Methods in Natural Language Processing (EMNLP), pages 1746-1751, Doha, Qatar. Association for Computational Linguistics.

Diederik P Kingma and Jimmy Ba. 2014. Adam: A method for stochastic optimization. arXiv preprint arXiv:1412.6980.

Diederik P Kingma and Max Welling. 2013. Autoencoding variational Bayes. arXiv preprint arXiv:1312.6114.

Jens Lehmann, Robert Isele, Max Jakob, Anja Jentzsch, Dimitris Kontokostas, Pablo N Mendes, Sebastian Hellmann, Mohamed Morsey, Patrick Van Kleef, Sören Auer, et al. 2015. DBpedia-a large-scale, multilingual knowledge base extracted from Wikipedia. Semantic Web, 6(2):167-195.

Bohan Li, Hao Zhou, Junxian He, Mingxuan Wang, Yiming Yang, and Lei Li. 2020. On the sentence embeddings from pre-trained language models. arXiv preprint arXiv:2011.05864.

Yinhan Liu, Myle Ott, Naman Goyal, Jingfei Du, Mandar Joshi, Danqi Chen, Omer Levy, Mike Lewis, Luke Zettlemoyer, and Veselin Stoyanov. 2019. ROBERTA: A robustly optimized BERT pretraining approach. arXiv preprint arXiv:1907.11692.

Sebastian Nowozin, Botond Cseke, and Ryota Tomioka. 2016. F-GAN: Training generative neural samplers using variational divergence minimization. arXiv preprint arXiv:1606.00709.

Aaron van den Oord, Yazhe Li, and Oriol Vinyals. 2018. Representation learning with contrastive predictive coding. arXiv preprint arXiv:1807.03748.

Zijing Ou, Qinliang Su, Jianxing Yu, Bang Liu, Jingwen Wang, Ruihui Zhao, Changyou Chen, and Yefeng Zheng. 2021. Integrating semantics and neighborhood information with graph-driven generative models for document retrieval. arXiv preprint arXiv:2105.13066. 
Jeffrey Pennington, Richard Socher, and Christopher D Manning. 2014. GloVe: Global vectors for word representation. In Proceedings of the 2014 Conference on Empirical Methods in Natural Language Processing, pages 1532-1543.

Zexuan Qiu, Qinliang Su, Zijing Ou, Jianxing Yu, and Changyou Chen. 2021. Unsupervised hashing with contrastive information bottleneck. arXiv preprint arXiv:2105.06138.

Nils Reimers and Iryna Gurevych. 2019. Sentencebert: Sentence embeddings using siamese bertnetworks. arXiv preprint arXiv:1908.10084.

Danilo Jimenez Rezende, Shakir Mohamed, and Daan Wierstra. 2014. Stochastic backpropagation and approximate inference in deep generative models. arXiv preprint arXiv: 1401.4082.

Ruslan Salakhutdinov and Geoffrey Hinton. 2009. Semantic hashing. International Journal of Approximate Reasoning, 50(7):969-978.

Dinghan Shen, Qinliang Su, Paidamoyo Chapfuwa, Wenlin Wang, Guoyin Wang, Lawrence Carin, and Ricardo Henao. 2018. NASH: Toward end-to-end neural architecture for generative semantic hashing. arXiv preprint arXiv: 1805.05361.

Karl Stratos and Sam Wiseman. 2020. Learning discrete structured representations by adversarially maximizing mutual information. In International Conference on Machine Learning, pages 9144-9154. PMLR.

Fan-Yun Sun, Jordan Hoffmann, Vikas Verma, and Jian Tang. 2019. InfoGraph: Unsupervised and semisupervised graph-level representation learning via mutual information maximization. arXiv preprint arXiv:1908.01000.

Fangbo Tao, Chao Zhang, Xiusi Chen, Meng Jiang, Tim Hanratty, Lance Kaplan, and Jiawei Han. 2018. Doc2Cube: Allocating documents to text cube without labeled data. In IEEE International Conference on Data Mining, pages 1260-1265. IEEE.

Laurens van der Maaten and Geoffrey Hinton. 2008 Visualizing data using t-SNE. Journal of Machine Learning Research, 9(86):2579-2605.

Ashish Vaswani, Noam Shazeer, Niki Parmar, Jakob Uszkoreit, Llion Jones, Aidan N Gomez, Lukasz Kaiser, and Illia Polosukhin. 2017. Attention is all you need. arXiv preprint arXiv:1706.03762.

Petar Velickovic, William Fedus, William L Hamilton, Pietro Liò, Yoshua Bengio, and R Devon Hjelm. 2019. Deep graph InfoMax. In International Conference on Learning Representations (Poster).

Thomas Wolf, Lysandre Debut, Victor Sanh, Julien Chaumond, Clement Delangue, Anthony Moi, Pierric Cistac, Tim Rault, Rémi Louf, Morgan Funtowicz, and Jamie Brew. 2019. Huggingface's transformers: State-of-the-art natural language processing. CoRR, abs/1910.03771.
Fanghua Ye, Jarana Manotumruksa, and Emine Yilmaz. 2020. Unsupervised few-bits semantic hashing with implicit topics modeling. In Conference on Empirical Methods in Natural Language Processing (Findings), volume 20, pages 2566-2575. Association for Computational Linguistics.

Xiang Zhang, Junbo Zhao, and Yann LeCun. 2015. Character-level convolutional networks for text classification. arXiv preprint arXiv:1509.01626.

Lin Zheng, Qinliang Su, Dinghan Shen, and Changyou Chen. 2020. Generative semantic hashing enhanced via Boltzmann machines. arXiv preprint arXiv:2006.08858. 